\title{
New XMM-Newton observations of supernova remnants in the Small Magellanic Cloud
}

\author{
M. D. Filipović ${ }^{1,2}$, F. Haberl ${ }^{1}$, P. F. Winkler ${ }^{3}$, W. Pietsch ${ }^{1}$, J. L. Payne ${ }^{4}$, E. J. Crawford ${ }^{2}$, A. Y. De Horta ${ }^{2}$, \\ F. H. Stootman ${ }^{2}$, and B. E. Reaser ${ }^{5}$
}

1 Max-Planck-Institut für extraterrestrische Physik, Giessenbachstrasse, 85741 Garching, Germany e-mail: [fwh; wnp] ampe .mpg.de

2 University of Western Sydney, Locked Bag 1797, Penrith South DC, NSW 1797, Australia

e-mail: [m.filipovic;e.crawford;a.dehorta;f.stootman]@uws.edu.au

3 Department of Physics, Middlebury College, Middlebury, VT 05753, USA

e-mail: winkler@middlebury.edu

4 Centre for Astronomy, James Cook University, Townsville QLD, 4811, Australia

e-mail: Jeffrey.Payne@jcu.edu.au

5 Department of Physics and Astronomy, Swarthmore College, 500 College avenue, Swarthmore, PA 19081, USA

Received 25 February 2008 / Accepted 20 April 2008

\section{ABSTRACT}

\begin{abstract}
Context. A complete overview of the supernova remnant (SNR) population is required to investigate their evolution and interaction with the surrounding interstellar medium in the Small Magellanic Cloud (SMC).

Aims. Recent XMM-Newton observations of the SMC cover three known SNRs (DEM S5, SNR B0050-72.8, and SNR B0058-71.8), which are poorly studied and are X-ray faint. We used new multi-frequency radio-continuum surveys and new optical observations at $\mathrm{H}_{\alpha}$, [S II] and [O III] wavelengths, in combination with the X-ray data, to investigate their properties and to search for new SNRs in the SMC

Methods. We used X-ray source selection criteria and found one SMC object with typical SNR characteristics (HFPK 334), that was initially detected by ROSAT. We analysed the X-ray spectra and present multi-wavelength morphological studies of the three SNRs and the new candidate.

Results. Using a non-equilibrium ionisation collisional plasma model, we find temperatures $k T$ around $0.18 \mathrm{keV}$ for the three known remnants and $0.69 \mathrm{keV}$ for the candidate. The low temperature, low surface brightness, and large extent of the three remnants indicates relatively large ages. The emission from the new candidate (HFPK 334) is more centrally peaked and the higher temperature suggests a younger remnant. Our new radio images indicate that a pulsar wind nebulae (PWN) is possibly associated with this object. Conclusions. The SNRs known in the SMC show a variety of morphological structures that are relatively uncorrelated in the different wavelength bands, probably caused by the different conditions in the surrounding medium with which the remnant interacts.
\end{abstract}

Key words. galaxies: Magellanic Clouds - ISM: supernova remnants

\section{Introduction}

The study of X-ray supernova remnants (SNRs) in nearby galaxies is of major interest for understanding both the X-ray output of more distant galaxies and the processes that occur on local interstellar scales within our own Galaxy. Unfortunately, the distances to many Galactic remnants are uncertain by a factor of 2 , leading to a factor of 4 uncertainty in luminosity and of 5.5 in the calculated energy release of the initiating supernova (SN). At a distance of $\sim 60 \mathrm{kpc}$ (Hilditch et al. 2005), the Small Magellanic Cloud (SMC) is one of the prime targets for the astrophysical research of extragalactic objects, including SNRs. This is because these remnants are located at a known distance, yet close enough to allow a detailed analysis of them.

SNRs reflect a major process in the elemental enrichment of the interstellar medium (ISM). Multiple supernova explosions close together generate super-bubbles typically hundreds of parsecs in extent. Both SNRs and super-bubbles are prime drivers controlling the morphology and the evolution of the ISM. Their properties are therefore crucial for a full understanding of the galactic matter cycle.
Previous X-ray surveys of the SMC have been undertaken using the Einstein (Wang \& Wu 1992), ROSAT (Haberl et al. 2000), and ASCA (Yokogawa et al. 2003) satellites. These surveys revealed discrete $\mathrm{X}$-ray sources and a large-scale diffuse emission. In particular, the high sensitivity and the large field of view of the ROSAT PSPC instrument has provided the most comprehensive catalogue of discrete X-ray sources, 517 in an area of $\sim 18$ square degrees (Haberl et al. 2000). It also has revealed the existence of a hot thin plasma within the SMC ISM having temperatures between $10^{6}$ and $10^{7} \mathrm{~K}$ (Sasaki et al. 2002).

The XMM-Newton (Jansen et al. 2001) archive contains a number of observations in the direction of the SMC, and they mainly cover the bar and part of the eastern wing of the galaxy (Haberl \& Pietsch 2007; Haberl et al., in preparation). Typical limiting point-source luminosities of a few $10^{33} \mathrm{erg} \mathrm{s}^{-1}$ are reached with XMM-Newton and extended objects like SNRs can be easily resolved ${ }^{1}$.

Today a total number of eighteen classified SNRs are known in the SMC, which are listed in Table 1 (Filipović et al. 2005;

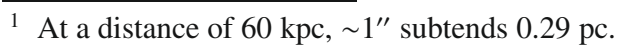


Table 1. SNRs and SNR candidates in the SMC. The radio spectral index $(\alpha)$ is taken from Filipović et al. (2005) and [S II]/H $\alpha$ ratio from Payne et al. (2007).

\begin{tabular}{|c|c|c|c|c|c|c|c|c|}
\hline No & SNR Name & $\begin{array}{c}\text { RA } \\
(\mathrm{J} 2000)\end{array}$ & $\begin{array}{c}\text { Dec } \\
(\mathrm{J} 2000)\end{array}$ & $\begin{array}{c}D \\
{[\mathrm{pc}]}\end{array}$ & $\begin{array}{c}S_{1 \mathrm{GHz}} \\
{[\mathrm{Jy}]}\end{array}$ & $\overline{\bar{\alpha}}$ & $\overline{[\mathrm{S}} \mathrm{II}] / \mathrm{H} \alpha$ & Other Names \\
\hline 1 & DEM S5 & 004100.1 & -733630.0 & $68.4 \times 62.6$ & 0.057 & -0.9 & 0.8 & HFPK 530 \\
\hline 2 & DEM S32 & 004637.6 & -730756.6 & $40.7 \times 46.5$ & 0.036 & -0.6 & 0.4 & Part of N S19, WW 15 \\
\hline 3 & J0047-7306 & 004728.6 & -730615.5 & $32.0 \times 8.7$ & 0.035 & -0.1 & 1.2 & part of N S19 \\
\hline 4 & HFPK 419 & 004736.5 & -730914.0 & $44.2 \times 33.7$ & 0.215 & -0.6 & 0.4 & part of N S19, HFPK 419 \\
\hline 5 & IKT 2 & 004716.6 & -730843.5 & $29.1 \times 32$ & 0.500 & -0.6 & 0.4 & N S19, DEM S32, WW 16, HFPK 413 \\
\hline 6 & IKT 4 & 004820.6 & -731939.6 & $45.1 \times 34.9$ & 0.176 & -1.0 & 0.4 & N S24, WW 21, DEM S42, HFPK 454 \\
\hline 7 & IKT 5 & 004907.7 & -731435.0 & 48.3 & 0.034 & -0.6 & 0.7 & DEM S49, WW 22, HFPK 437 \\
\hline 8 & IKT 6 & 005106.7 & -73 2121.4 & 42.2 & 0.137 & -0.7 & 0.5 & 1E0049.4-7339, WW 24, HFPK 461 \\
\hline 9 & B0050-72.8 & 005236.9 & -723718.5 & 42 & 0.273 & -1.0 & 0.4 & N S50, DEM S68, WW 30, HFPK 285 \\
\hline 10 & N S66D & 005800.0 & -721101.4 & 58.2 & 0.057 & 0.0 & 0.4 & \\
\hline 11 & IKT 16 & 005817.8 & -721807.4 & 58 & 0.091 & -0.7 & 0.5 & WW 42 \\
\hline 12 & IKT 18 & 005927.7 & -721009.8 & 40.7 & 0.501 & -0.8 & 1.7 & N S66, NGC 346, WW 44, HFPK 148 \\
\hline 13 & B0058-71.8 & 010023.9 & -713341.1 & 61 & 0.241 & -0.8 & [O III] & DEM S108, HFPK 45 \\
\hline 14 & IKT 21 & 010317.0 & -720945.0 & 20.9 & 0.123 & -0.5 & 0.5 & N S76C, WW 50 \\
\hline 15 & 1E0102-723 & 010401.2 & -720152.3 & $5.8 \times 10.5$ & 0.363 & -0.6 & [O III $]$ & N S76, IKT 22, WW 51, HFPK 107 \\
\hline 16 & IKT 23 & 010504.2 & -722310.5 & 55.9 & 0.112 & -0.7 & [O III] & DEM S125, WW 52, HFPK 217 \\
\hline 17 & DEM S128 & 010524.7 & -720920.4 & 37.8 & 0.060 & -0.5 & 0.6 & IKT 24, WW 53, HFPK 145 \\
\hline 18 & IKT 25 & 010627.5 & -720534.5 & $32 \times 23$ & 0.014 & -0.7 & 0.4 & DEM S131, WW 54, HFPK 125 \\
\hline 19 & HFPK 334 & 010329.5 & -724723.2 & 17.5 & 0.025 & -0.5 & - & HFPK 334 \\
\hline 20 & B0113-729 & 011333.8 & -731704.4 & 21.8 & 0.108 & -0.6 & 0.4 ? & N S83C, DEM S147, HFPK 448 \\
\hline
\end{tabular}

Payne et al. 2007; van der Heyden et al. 2004; Dickel et al. 2001; Mathewson et al. 1984; Mills et al. 1982; Hughes \& Smith 1994; Nazé et al. 2004, and references therein). Thirteen of the remnants were covered by early XMM-Newton observations and a comprehensive study was presented by van der Heyden et al. (2004). Archival data and our own proprietary observations (from the AO5 and AO6 observing periods) cover three more faint remnants. The inspection of the EPIC images also revealed a new SNR candidate.

Recently, radio-continuum observations at 20, 13, 6, and $3 \mathrm{~cm}(1420,2370,4800$, and $8640 \mathrm{MHz})$ with the Australia Telescope Compact Array (ATCA) were performed to study radio SNRs in the direction of the SMC (Filipović et al. 2002, 2005, and references therein). Follow-up optical spectroscopy allowed us to confirm one candidate SNR and study 11 other known remnants. Line intensity ratios provided rough estimates of the average SMC "metal" abundance, supporting the idea that these ratios are related more to the ISM than to SNR ejecta (Payne et al. 2007).

The present paper is organised as follows: Sect. 2 outlines our selection and analysis of the XMM-Newton data. We also discuss complementary radio and optical data in greater detail. In Sect. 3, a multi-frequency analysis of each remnant or candidate is presented and a discussion of SNRs not detected in any X-ray observations. Our conclusions are given in Sect. 4.

\section{Observational data}

\subsection{XMM-Newton observations of SMC fields}

In order to investigate the X-ray source population of the SMC, Haberl \& Pietsch (2007) started a systematic analysis of the available XMM-Newton data. This included their own proprietary data together with data from the public archive. Only observations performed with the EPIC-PN instrument (Strüder et al. 2001) in Large Window (LW), Full Frame (FF) or extended Full Frame (eFF) imaging CCD readout mode were considered. The data were analysed using the analysis package XMMSAS version 7.0.0.
The XMM-Newton observations cover three SNRs which have not yet been studied in detail in X-rays due to their faintness: DEM S5 (HFPK 530), SNR B0050-72.8 (DEM S68; HFPK 285) and SNR B0058-71.8 (DEM S108; HFPK 45). All three were detected in ROSAT PSPC data (Haberl et al. 2000, with catalogue entry given by their HFPK number) and were not yet observed by XMM-Newton at the time of the work of van der Heyden et al. (2004). Another ROSAT PSPC source HFPK 334, (with evidence for spatial extent in the ROSAT data and a possible radio counterpart) shows X-ray colours typical of SNRs and we suggest it as new candidate SNR.

In the following, we investigate the properties of the three X-ray faint SNRs and the new candidate SNR HFPK 334. For morphology studies the XMM-Newton EPIC images in different energy bands were compared with radio-continuum and optical images (see below). For X-ray spectral analysis EPIC spectra were extracted for PN (single + double pixel events, corresponding to a PATTERN 0-4 selection) and MOS (Turner et al. 2001, PATTERN 0-12), excluding bad CCD pixels and columns (FLAG 0). These were fitted simultaneously, allowing only a renormalisation factor to account for cross-calibration uncertainties between the instruments.

We used $\mathrm{XSPEC}^{2}$ version 11.3.2p for spectral modelling. To account for photo-electric absorption by interstellar gas, two hydrogen column densities were used. The first represents the foreground absorption in the Milky Way, fixed at $6 \times 10^{20} \mathrm{~cm}^{-2}$ assuming elemental abundances of Wilms et al. (2000). The second considers the absorption in the SMC (with metal abundances set to 0.2 solar as typical of the SMC; Russell \& Dopita 1992). The statistical quality of the spectra was sufficient to fit onecomponent thermal plasma emission models. We used a singletemperature non-equilibrium ionisation collisional plasma (NEI) model (in XSPEC, see Borkowski et al. 2001, and references therein) with metal abundances fixed to 0.2 solar, yielding acceptable $\chi^{2}$ values.

\footnotetext{
${ }^{2}$ http://heasarc.gsfc.nasa.gov/docs/xanadu/xspec/
} 

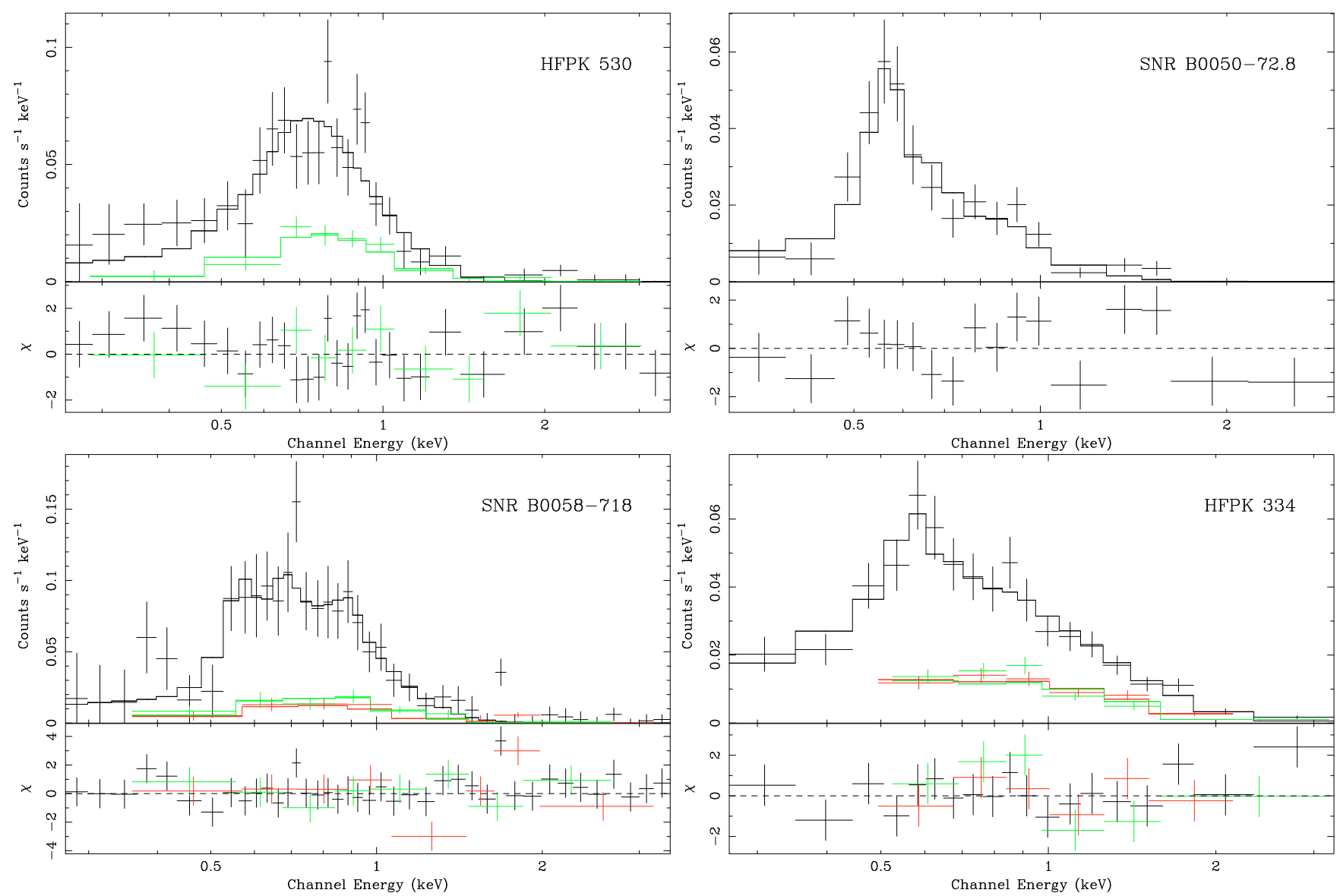

Fig. 1. EPIC spectra of supernova remnants in the SMC. From top left to bottom right: a) SNR B0039-7353 = HFPK 530, b) SNR B0050-72.8, c) SNR B0058-71.8 and d) the candidate SNR HFPK 334. The best fits using a single-temperature NEI model are plotted as histograms (black: PN, red: MOS1, green: MOS2).

Table 2. Spectral fits to EPIC spectra of SNRs with an absorbed NEI model.

\begin{tabular}{lccccccccc}
\hline \hline Source & $\begin{array}{c}\text { PN exp. } \\
{\left[10^{3} \mathrm{~s}\right]}\end{array}$ & $\begin{array}{c}\text { SMC } N_{\mathrm{H}} \\
{\left[10^{21} \mathrm{~cm}^{-2}\right]}\end{array}$ & $\begin{array}{c}k T \\
{[\mathrm{keV}]}\end{array}$ & $\begin{array}{c}\tau \\
{\left[10^{8} \mathrm{~s} \mathrm{~cm}^{-3}\right]}\end{array}$ & $\begin{array}{c}F_{\mathrm{x}} \\
{\left[\mathrm{erg} \mathrm{cm}^{-2} \mathrm{~s}^{-1}\right]}\end{array}$ & $\begin{array}{c}L_{\mathrm{x}} \\
{\left[\mathrm{erg} \mathrm{s}^{-1}\right]}\end{array}$ & $\begin{array}{c}\text { EM } \\
{\left[\mathrm{cm}^{-3}\right]}\end{array}$ & $\begin{array}{c}\chi_{r}^{2} \\
\text { d.o.f. }\end{array}$ \\
\hline HFPK 530 & 17.7 & $7.7_{-1.9}^{+7.7}$ & $0.18 \pm 0.06$ & $2_{-1}^{+19}$ & $8.4 \times 10^{-14}$ & $1.8_{-0.9}^{+40} \times 10^{36}$ & $1.1_{-0.5}^{+9.0} \times 10^{60}$ & 1.12 & 34 \\
SNR B0050-72.8 & 27.1 & $7.4_{-2.4}^{+3.0}$ & $0.19 \pm 0.08$ & $17_{-6}^{+18}$ & $6.7 \times 10^{-14}$ & $2.7_{-1.8}^{+4.4} \times 10^{36}$ & $3.7_{-2.7}^{+7.3} \times 10^{59}$ & 1.52 & 14 \\
SNR B0058-71.8 & 11.9 & $12.0 \pm 5.8$ & $0.17 \pm 0.07$ & $17_{-13}^{+9}$ & $8.4 \times 10^{-14}$ & $1.1_{-1.0}^{+4.9} \times 10^{37}$ & $1.9_{-1.6}^{+9.2} \times 10^{60}$ & 1.17 & 50 \\
HFPK 334 & 17.7 & $2.7 \pm 0.9$ & $0.69 \pm 0.12$ & $4.8 \pm 1.2$ & $1.3 \times 10^{-13}$ & $3.6_{-0.9}^{+1.3} \times 10^{35}$ & $3.1_{-0.5}^{+0.5} \times 10^{58}$ & 1.19 & 25 \\
\hline
\end{tabular}

The EPIC spectra of all four of these X-ray sources are plotted in Fig. 1 using their best-fit NEI models. The derived best fit model parameters (SMC absorption, temperature $k T$ and ionisation time scale $\tau$ ) are summarised in Table 2 together with inferred fluxes and luminosities. Flux and luminosity are given for 0.2-2 keV, determined from the EPIC-PN spectrum (HFPK 530: MOS2). Intrinsic source luminosity with total $N_{\mathrm{H}}$ set to 0 , assuming a distance of $60 \mathrm{kpc}$ to the SMC. The relatively large errors in the SMC absorption leads to large uncertainties in the (absorption corrected) luminosities. Therefore, we give a luminosity range derived from fits with $\mathrm{SMC} N_{\mathrm{H}}$ fixed at the lower and upper confidence values. These very soft temperature estimates of $N_{\mathrm{H}}$ ranging from 7 to $12 \times 10^{21}$ are consistent with very soft emission from older SNRs, obscured by heavy foreground extinction. Similarly we derive a confidence range for the emission measure EM. The spectra of the three SNRs DEM S5, SNR B0050-72.8 and SNR B0058-71.8 are characterised by a high absorption and a low temperature. Considering their large extent and low surface brightness, they are most likely older remnants. The spectral analysis of the SNR candidate HFPK 334 yields a higher temperature, similar to what is found for most of the known SMC SNRs.

\subsection{The ATCA radio-continuum surveys of the SMC}

We used all available radio-continuum images of the SMC, including mosaics and specific ATCA target pointings. These are composed of observations at 5 radio frequencies having moderate resolution at $36 \mathrm{~cm}(843 \mathrm{MHz})$ (MOST; Turtle et al. 1998) and 20, 13, 6 and $3 \mathrm{~cm}$ (ATCA; Filipović et al. 2002; Dickel et al. 2001). In addition, we use special lower-resolution ATCA observations towards DEM S5 and SNR B0058-71.8 (DEM S108), as described in Filipović et al. (2005). These moderate resolution observations $\left(>12^{\prime \prime}\right)$ allow a reasonable insight into the nature of these objects. 

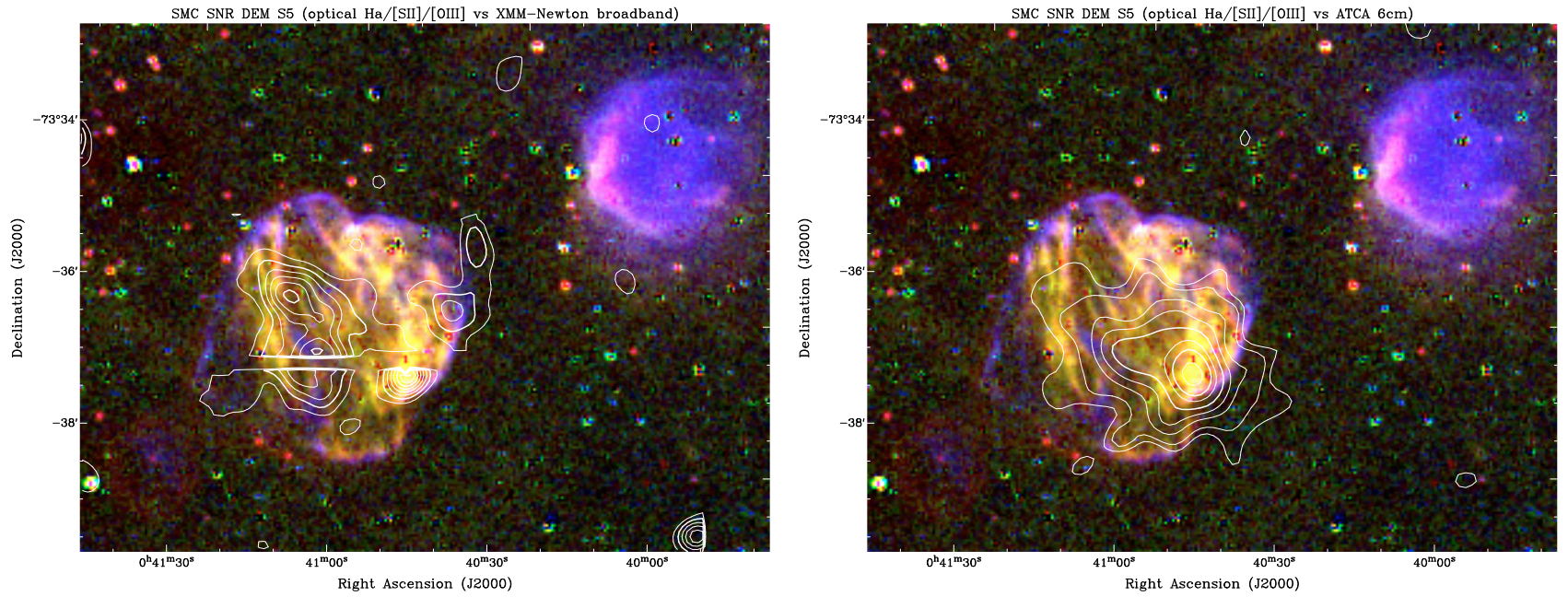

Fig. 2. A composite optical image ( $\mathrm{RGB}=\mathrm{H}_{\alpha}$, [S II] and [O III], respectively, all continuum-subtracted) of SMC SNR DEM S5 overlaid with (A) XMM-Newton broad-band contours and (B) ATCA 6-cm contours. The X-ray contours are 15, 20, 25, 30, 35, 40, and 50 $\times 10^{-6} \mathrm{ct} \mathrm{s}^{-1} \mathrm{pix}^{-1}$, and the radio-continuum contours are 2, 3, 5, 7, 10, 15, 20 and $25 \mathrm{mJy} /$ beam. Note that the [O III] emission is brightest at the outside of the shell, closest to the expanding SNR shock, with $\mathrm{H}_{\alpha}$, [S II] lagging behind where the post-shock gas has cooled somewhat. The prominent bluish object at the upper right (north-west - centred at $\mathrm{RA}(\mathrm{J} 2000)=00^{\mathrm{h}} 40^{\mathrm{m}} 02.6^{\mathrm{s}}$ and $\left.\mathrm{Dec}(\mathrm{J} 2000)=-73^{\circ} 33^{\prime} 53^{\prime \prime}\right)$, with faint $\left[\mathrm{O}\right.$ III] inside and $\mathrm{H}_{\alpha}$ around much of the ill-defined periphery, is a photo-ionised region. Another, much fainter region of [O III] emission is just south-east of DEM S5 $\left(\mathrm{RA}(\mathrm{J} 2000)=00^{\mathrm{h}} 41^{\mathrm{m}} 32.7^{\mathrm{s}}\right.$ and $\left.\operatorname{Dec}(\mathrm{J} 2000)=-73^{\circ} 38^{\prime} 22^{\prime \prime}\right)$.

The ATCA observations of HPFK 334 were made on 15th November 2007 using array configuration EW3673, and on 14th January 2008 using array configuration $6 \mathrm{~A}^{3}$, at wavelengths of 6 and $3 \mathrm{~cm}$. The observations were done in so called "snap-shot" mode totalling $\sim 4$ h of integration over a 12-h period with each array. Source 1934-638 was used for primary calibration and 0252-712 for secondary calibration. The MIRIAD (Sault \& Killeen 2006) and KARMA (Gooch 2006) software packages were used for reduction and analysis. At 6 and $3 \mathrm{~cm}$, the image resolution is, respectively, $21^{\prime \prime} \times 20^{\prime \prime}$ and $12^{\prime \prime} \times 11^{\prime \prime}$, and the rms noise is estimated to be $0.13 \mathrm{mJy} /$ beam and $0.14 \mathrm{mJy} / \mathrm{beam}$.

\subsection{The MCELS optical surveys of the SMC}

The Magellanic Cloud Emission Line Survey (MCELS) was carried out from the $0.6 \mathrm{~m}$ University of Michigan/CTIO Curtis Schmidt telescope, equipped with a SITE $2048 \times 2048$ CCD, which gave a field of $1.35^{\circ}$ at a scale of $2.4^{\prime \prime}$ pixel $^{-1}$. Both the LMC and SMC were mapped in narrow bands corresponding to $\mathrm{H}_{\alpha}$, [O III $](\lambda=5007 \AA)$, and [S II] $(\lambda=6716,6731 \AA)$, plus matched red and green continuum bands that are used primarily to subtract most of the stars from the images to reveal the full extent of the faint diffuse emission. A $4.5^{\circ} \times 3.5^{\circ}$ region, most of the SMC, was covered in 69 overlapping fields, offset so that every point was included in at least four different fields. All the data have been flux-calibrated and assembled into mosaic images, small sections of which are shown in Figs. 2-4. (Further details regarding the MCELS are given by Smith et al. (2006), Winkler et al. (in preparation) and at http://WwW . ctio. noao. edu/mcels/.)

\section{Results and discussion of individual objects}

\subsection{The SMC SNR DEM S5 (HFPK 530; J004100-733648)}

The emission nebulae DEM S5 (Davies et al. 1976) was originally classified as an SNR candidate in our ROSAT survey

\footnotetext{
3 http://www narrabri. atnf.csiro.au/observing/configs. html
}

(Haberl et al. 2000). Optical spectroscopy and low-resolution radio-continuum analysis of this object can be found in Payne et al. (2007) and Filipović et al. (2005). We estimate DEM S5's radio spectral index ${ }^{4}$ to be -0.9 and its optical $[\mathrm{S} \mathrm{II}] / \mathrm{H}_{\alpha}$ ratio about 0.8 .

The remnant is $\sim 210^{\prime \prime}(60 \mathrm{pc}$ ) in size, with a complex shelllike optical structure shown in Fig. $2\left(\mathrm{RGB}=\mathrm{H}_{\alpha} /[\mathrm{S} \mathrm{II}] /[\mathrm{O}\right.$ III $]$, all with a matched continuum subtracted). The optical shell could comprise of two intersecting ring systems, one elongated northsouth, and the other (most prominent in [O III]) elongated eastwest. However, we acknowledge that this needs to be confirmed by more precise kinematic analysis. The outer periphery of almost the entire shell is marked by [O III] emission, characteristic of excitation by shocks with $v \gtrsim 100 \mathrm{~km} \mathrm{~s}^{-1}$, with stronger $\mathrm{H}_{\alpha}$ and $[\mathrm{S}$ II] emission from the cooling regions behind the shock. Our new XMM-Newton image (Fig. 2) shows multiple peaks around and within the optical shell. However, both the X-ray and the radio-continuum images have a slightly smaller extent than that seen optically, most likely due to lower resolution and sensitivity. Nevertheless, DEM S5 ranks among the largest SNRs in the SMC. EPIC spectra of the remnant were extracted for PN and MOS2 (Fig. 1a) and are typical of SNRs; the MOS1 detector did not cover the source.

We note that the peak emission in the X-ray and radiocontinuum images coincides with a small region, $\sim 16^{\prime \prime}$ in size and centred at $\mathrm{RA}(\mathrm{J} 2000)=00^{\mathrm{h}} 40^{\mathrm{m}} 47.7^{\mathrm{s}}$ and $\operatorname{Dec}(\mathrm{J} 2000)=$ $-73^{\circ} 37^{\prime} 03^{\prime \prime}$, where the $\mathrm{H}_{\alpha}$ and [S II] emission is also brightest. This may simply be a dense cloud that has been overrun by the SNR shock, but the X-ray peak is not spatially resolved and has a harder spectrum than the rest of the remnant, so we cannot exclude a line-of-sight association with an active galaxy or quasar.

\subsection{The SMC SNRB0050-72.8 (DEM S68; HFPK 285; J005240-723820)}

The complex nebulae SNR B0050-72.8 was identified as a SNR by Mathewson et al. (1984) and Mills et al. (1982) based on

${ }^{4}$ Defined as $\alpha$, where $S_{v} \propto v^{\alpha}$. 

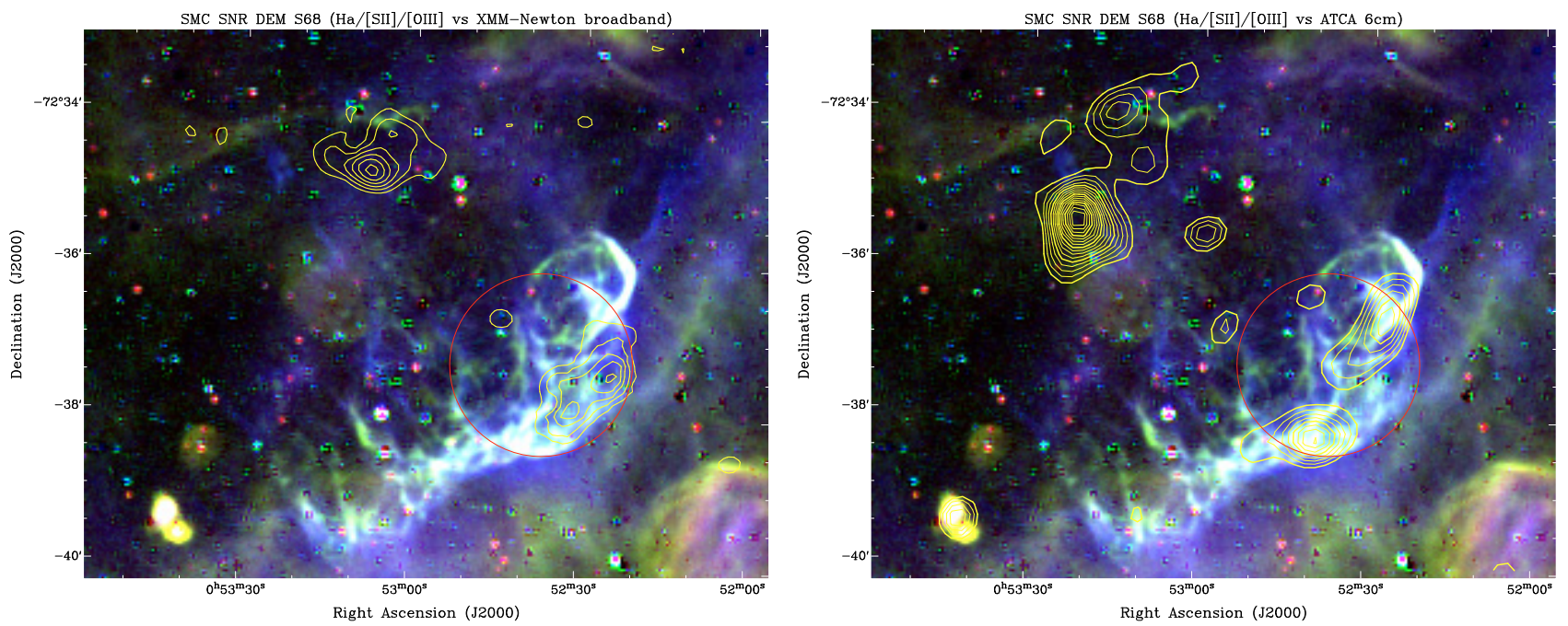

Fig. 3. A composite optical image (RGB - $\mathrm{H}_{\alpha}$, [S II] and [O III]) of SMC SNR B0050-72.8 overlaid with (A) XMM-Newton broad-band contours and (B) ATCA 6-cm contour map. X-ray contours are 15, 20, 25, 30, 35, and $40 \times 10^{-6} \mathrm{ct} \mathrm{s}^{-1} \mathrm{pix}^{-1}$. Radio-continuum contours are from 1 to $5 \mathrm{mJy} /$ beam in steps of $0.25 \mathrm{mJy} / \mathrm{beam}$. The red circle indicates the position and extent of the remnant. The bright object in the lower left corner is the compact H II region DEM S69.

optical and radio-continuum (MOST) detections. They suggest this remnant has a steep (non-thermal) radio-continuum spectrum and is most likely an older SNR since it has a diameter of $\sim 57$ pc. Filipović et al. (2005) confirmed its radio nonthermal nature $(\alpha=-1.0)$ and Payne et al. (2007) estimated a $[\mathrm{S} \mathrm{II}] / \mathrm{H}_{\alpha}$ ratio of 0.4 . A ROSAT detection was reported by Haberl et al. (2000) who designated it HFPK 285.

From our optical images we observe a number of ring structures, with a distinctive loop, that may correspond to the radio and X-ray emissions, centred at RA(J2000) $=00^{\mathrm{h}} 52^{\mathrm{m}} 36.9^{\mathrm{s}}$ and $\operatorname{Dec}(\mathrm{J} 2000)=-72^{\circ} 37^{\prime} 18.5^{\prime \prime}$. The remnant's optical emission is brighter to the south and west with no emission in the north-east region. Two bright radio-continuum peaks (Fig. 3) indicate radio emission which coincides with the optical peaks.

We estimate SNR B0050-72.8's diameter as $145 \pm 10^{\prime \prime}$ (42 pc), somewhat smaller than suggested by Mathewson et al. (1984). This is most likely due to the complexity of the region which is superimposed by a $\sim 300^{\prime \prime}$ ( $87 \mathrm{pc}$ ) elongated shell (or bubble).

SNR B0050-72.8 was covered by three XMM-Newton observations, in all cases at the rim of the EPIC FOV. The EPIC image shows extended emission at the south-west rim of the optical emission. However, the peak X-ray emission doesn't coincide with either the radio-continuum or the optical emission. The EPIC image also shows extended emission in the northeast, about $1^{\prime}$ outside of the optical emission. The similar X-ray colours of the two emission components suggest they are either both related to the larger SNR (bubble) or that the north-east source is an independent new SNR (Fig. 3). We could not find any obvious radio-continuum or optical emission corresponding to this X-ray source.

An alternate explanation is an SNR in a larger cavity or bubble. In this model, the radio and X-ray to the NE and in the red circle may just be the brightest peaks in a larger shell of emission. This larger bubble in the centre of Fig. 3.

\subsection{The SMC SNRB0058-71.8 (DEM S108; HFPK 45; J010024-713336)}

This remnant was originally classified as an SNR by Mathewson et al. (1984) and Mills et al. (1982), based on both optical and radio-continuum (MOST) detections. It is centered at $\operatorname{RA}(\mathrm{J} 2000)=01^{\mathrm{h}} 00^{\mathrm{m}} 24^{\mathrm{s}}, \operatorname{Dec}(\mathrm{J} 2000)=-71^{\circ} 33^{\prime} 36^{\prime \prime}$, near the southwest rim of the giant H II region DEM S108 which may be associated with the stellar cluster Brück 101 (Brück 1976). Optical images (Fig. 4) show a well-defined, possibly barrellike shell with the axis oriented near east-west. Diffuse emission, primarily [O III], is present throughout the interior, but this is part of the much larger DEM S108 shell, which may or may not be physically associated with the SNR. The bright star located very near the (projected) SNR center is Tyc 9138-1786-1, a foreground star in the Galaxy (Høg et al. 2000).

The 6-cm ATCA radio image shows a very well-defined elliptical shell for the SNR, measuring $230^{\prime \prime} \times 180^{\prime \prime}(67 \times 52 \mathrm{pc})^{5}$, that corresponds extremely well with the optical filaments. Our earlier radio studies (Filipović et al. 2005) confirmed its nonthermal nature, with spectral index $\alpha=-0.8$ ). Haberl et al. (2000) detected this source with the ROSAT PSPC (HFPK 45).

EPIC observations of SNR B0058-71.8 included $11.9 \mathrm{ks}$ (PN) and $13.5 \mathrm{ks}(\mathrm{MOS})$ exposures. From the resulting image, we found its X-ray diameter to be $150^{\prime \prime} \times 120^{\prime \prime}(43 \times 35 \mathrm{pc})$; significantly smaller than its optical and radio extent. We note that a higher background emission, when considered with its apparent older age ${ }^{6}$ based on its X-ray spectrum (Fig. 1c) and large optical/radio diameter, may account for this. Although a number of X-ray SNRs are centrally condensed (like this object), most have clear shells at radio and optical frequencies. Paradoxically, its [O III] dominated emission confuses this picture, since these SNRs tend to be younger. However, it is possible that the [O III] that fills the SNR is part of the much larger DEM S108 shell.

We used the XMM-Newton observation having the longest exposure time, to extract an EPIC-PN spectrum (Fig. 1c). This covered only the western portion of two emission regions.

\subsection{The SMC SNR candidate HFPK 334 (J010329-724723)}

We found a new candidate SNR based on its X-ray colour or hardness (Fig. 5). Listed as number 334 in the PSPC catalogue (Haberl et al. 2000), it co-identifies with an extended $13 / 6 \mathrm{~cm}$

\footnotetext{
5 We estimate that the error in the diameter is $<10^{\prime \prime}(3 \mathrm{pc})$.

${ }^{6} \mathrm{X}$-ray emission from SNRs tend to fade earlier since it is predominantly thermal.
} 

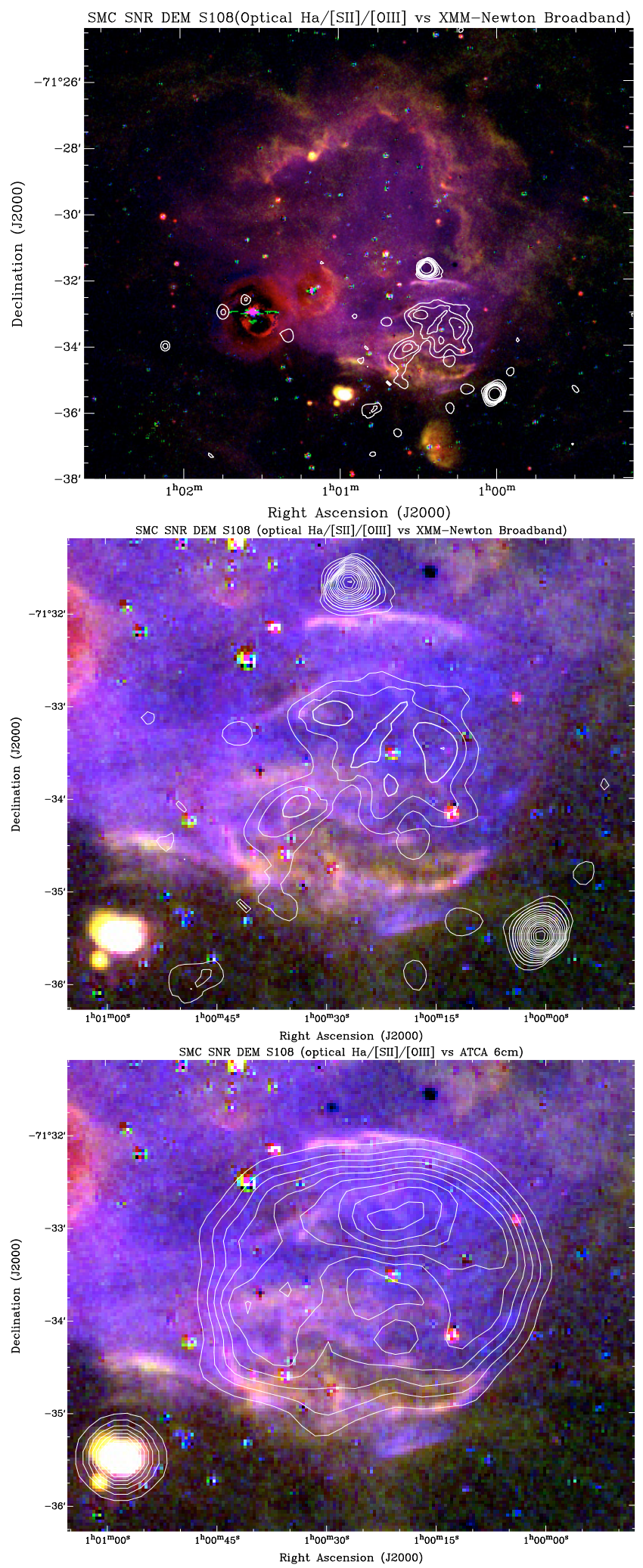

Fig. 4. A composite optical image (RGB $-\mathrm{H}_{\alpha}$, [S II] and [O III]) of SMC SNR B0058-71.8 overlaid with (A) XMM-Newton broad-band contours, showing the entire DEM S108 shell. An inset showing just the remnant overlaid with (B) XMM-Newton broad-band contours and (C) ATCA 6-cm contours. X-ray contours are 30, 35, 40, 45, 50, 70, and $150 \times 10^{-6} \mathrm{ct} \mathrm{s}^{-1} \mathrm{pix}^{-1}$. Radio-continuum contours are $0.5,1,1.5$, $2,2.5,3,3.5,4$, and $5 \mathrm{mJy} / \mathrm{beam}$. The bright object in the lower left corner is the compact $\mathrm{H}$ II region DEM S109.
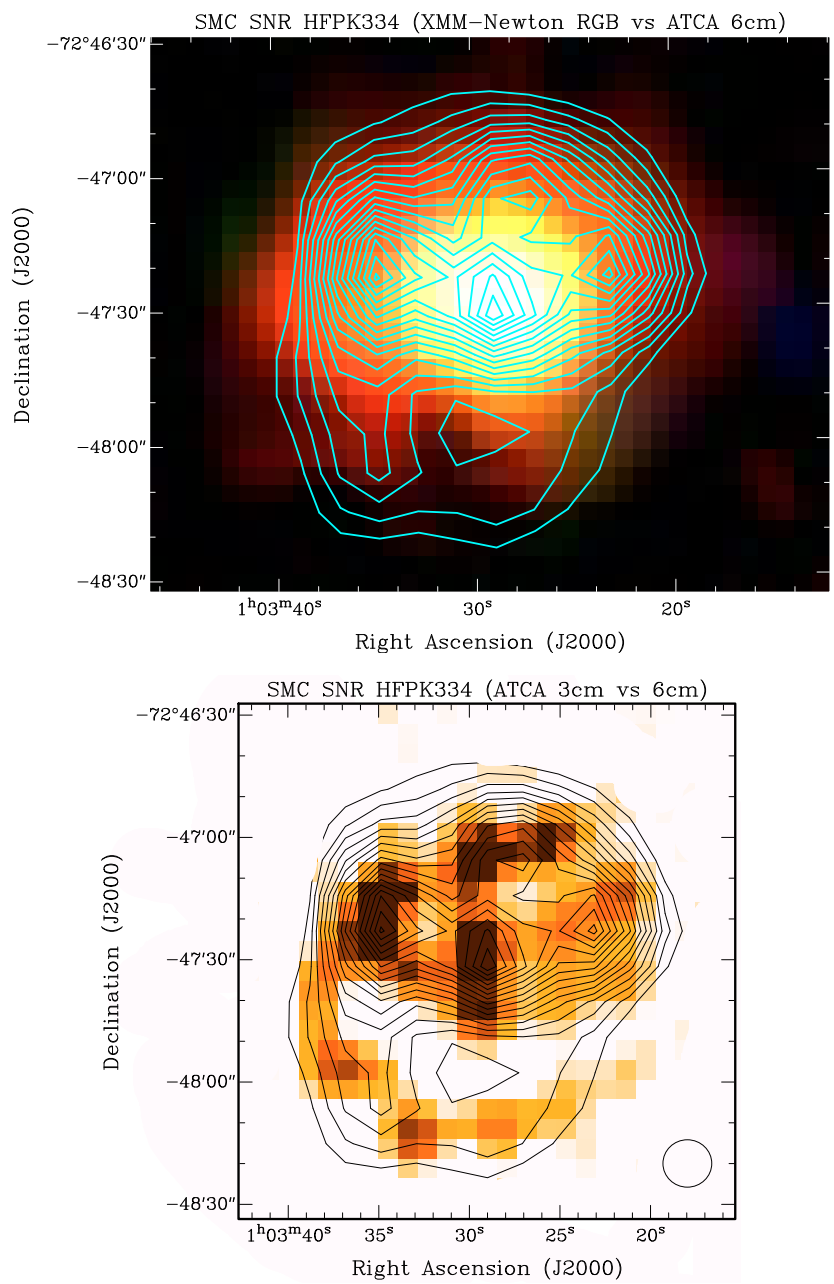

Fig. 5. (A) A composite XMM-Newton image (RGB - soft $(0.2-1.0 \mathrm{keV})$, medium (1.0-2.0 keV) and hard (2.0-4.5 keV)) of the SMC SNR HFPK 334 overlaid with ATCA 6-cm contour map. (B) ATCA 3-cm image overlaid with ATCA 6-cm contour map. The black circle represents the $3-\mathrm{cm}$ beam size of $12 \times 11^{\prime \prime}$. The radio-continuum contours at $6-\mathrm{cm}$ are from 0.27 to $3.0 \mathrm{mJy} /$ beam in $0.13 \mathrm{mJy} /$ beam steps.

radio source (Haberl et al. 2000). Following the naming convention used by van der Heyden et al. (2004) for SNRs detected in ROSAT data, we designate this candidate SNR HFPK 334. Its $\mathrm{X}$-ray spectrum, shown in Fig. 1d, was extracted using data from all three EPIC instruments.

Measured overall (total) integrated radio-continuum flux densities are 27.7, 22.1, 18.0, 14.3, and $8.6 \mathrm{mJy}^{7}$ at 36, 20, 13,6 , and $3 \mathrm{~cm}$, respectively. Using these values, we find a typical SNR spectral index $(\alpha)$ of $-0.5 \pm 0.1$. However, upon closer examination the total spectral index appears to be composed of two components, one from 36 to $6 \mathrm{~cm}$ with a value $\left(\alpha_{1}\right)$ of $-0.4 \pm 0.1$. The second is from 6 to $3 \mathrm{~cm}$ with a value $\left(\alpha_{2}\right)$ of $-0.9 \pm 0.1$. At 6 and $3 \mathrm{~cm}$, the source is resolved and extended with a diameter of $100^{\prime \prime} \times 80^{\prime \prime}(29 \times 23.2 \mathrm{pc})$ with an estimated error of $\pm 10^{\prime \prime}( \pm 2.9 \mathrm{pc})$. From Fig. 5 (at $\left.3 \mathrm{~cm}\right)$ we can see a bright point source in the centre of the proposed remnant. We measured the flux of this point source alone to be identical at 6 and $3 \mathrm{~cm}(2.4 \pm 0.4 \mathrm{mJy})$. This suggests that HFPK 334 central source is associated with the SNR shell, indicating a possibility of this object being a Pulsar Wind Nebula

\footnotetext{
7 An error is estimated to be $<10 \%$.
} 
(PWN). However, adding the longer baselines from the $6 \mathrm{~km}$ array (January 2008 observations) resolved out the SNR's extended emission and no point source could be associated with the remnant. The XMM-Newton data also shows an extended and possibly non-thermal source with a bright central peak, a similar situation to LMC SNR B0453-685 (Gaensler et al. 2003). Also, the two component flat radio spectral index of this point source $(\alpha=-0.0)$ seems to support this. Finally, we note that no reliable polarisation was detected at either 6 or $3 \mathrm{~cm}$ to a level of $10 \%$.

This object is thus unusual, as it is the first suspected PWN in the SMC. Furthermore, even very deep optical observations with various filters $\left(\left[\mathrm{O}_{\text {III }}\right],\left[\mathrm{S}_{\mathrm{II}}\right]\right.$ and $\mathrm{H}_{\alpha}$ ) failed to detect any optical emission from this proposed SNR. This would be the rare case of a SNR in the SMC without optical identification which even further indicate the complex nature of this object. Further high-resolution radio-continuum and X-ray studies would help to understand this object.

\subsection{SMC SNRs not detected in X-ray surveys}

Two objects classified as "definite" SMC SNRs are not detected in any X-ray surveys: SNR J004728-730601 (Dickel et al. 2001) and SNR J005800-721101 (Reid et al. 2006). In spite of the fact that both were covered by several XMM-Newton pointings, we could not detect any distinctive ${ }^{8} \mathrm{X}$-ray emission.

There are several other sources in the SMC classified as SNR candidates. These include J0051.9-7310 suggested by Dickel et al. (2001) and J011333-731704 suggested by Filipović et al. (2005), both based on radio-continuum images and tentative ROSAT detections. Although these regions contain point-like $\mathrm{X}$-ray emission that is suspicious of background objects, we did not find any extended X-ray emission to confirm these objects are SNRs. A very thin [S II] ring surrounding J011333731704 , possibly indicative of shocked regions, was the only optical emission detected.

Lack of X-ray emission from these SNR candidates is relatively surprising since some X-ray emission is generally expected. We acknowledge that this is a very complex issue, since we are dealing with the limiting sensitivities of very different instruments and at very different wavelengths coupled with observational parameters (e.g. foreground extinction and its variable effect on different wavelengths) and the variable nature of the sources being observed (large, evolved SNRs versus younger, smaller objects or objects evolving in diffuse fairly uniform ISM versus impacting nearby clumpy material). Payne et al. (2004) and Pannuti et al. (2007) found a number of optical/radio SNRs in NGC 300, M 81, M 101, NGC 2403, NGC 4736, NGC 6946 without any X-ray trace. They argue that the local ambient density plays a major role in addition to instrument sensitivity in these more distant galaxies.

Stupar et al. (in preparation) found 21 new Galactic SNRs using high resolution optical detection and spectroscopy, from the AAO/UKST SuperCOSMOS $\mathrm{H}_{\alpha}$ Survey (Parker et al. 2005). About half (10) of these could not be correlated with any X-ray or radio-continuum emission. They conclude that the local environment into which an individual SNR is propagating plays a very important role in this. Other factors include selection effects based on supernova type and survey sensitivity.

\footnotetext{
8 SNR J004728-730601 may exhibit a very low level X-ray emission; future XMM-Newton observations may reveal the true nature of this source.
}

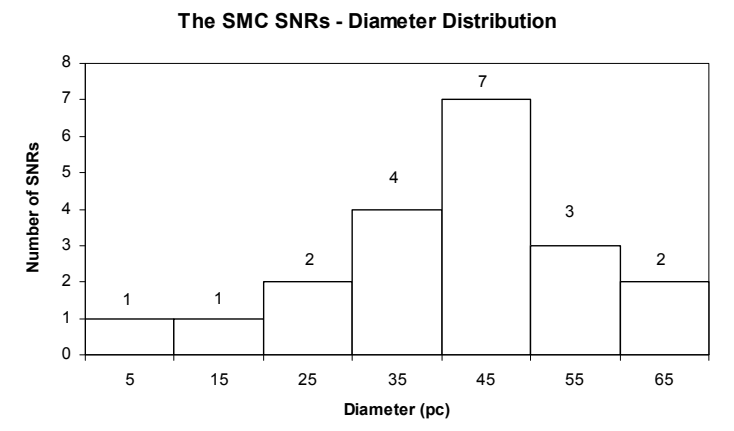

Fig. 6. A diameter distribution of the SMC SNRs and SNR candidates. We assume a distance to the SMC of $60 \mathrm{kpc}$.

\subsection{SMC SNRS diameter distribution}

With the new and moderate resolution surveys at various radio frequencies we can measure more precisely the diameters of the SMC SNRs and SNR candidates. We estimate the precision of our diameter measurements to be $<2^{\prime \prime}(<0.6 \mathrm{pc})$. Despite that some SMC SNRs (for example SNR B0058-71.8) exhibit different diameters across different part of the spectrum, we find that the majority of SNRs have consistent diameters at various frequencies.

In Table 1 (Col. 5) are a list of diameters for all SMC SNRs and SNR candidates. Figure 6 shows that the majority (7 out of 20) of the SMC SNRs have an average diameter of $\sim 45 \mathrm{pc}$. That indicates that most of the SMC SNRs might be in the adiabatic phase of evolution.

\section{Conclusions}

We have analysed our new and archival XMM-Newton observations in the direction to the SMC, discovering one new X-ray SNR candidate (HFPK 334) with strong indication for the existence of a PWN. In addition, we have examined three previously known SNRs (DEM S5, SNR B0050-72.8, and SNR B0058-71.8), expanding their X-ray emission details significantly. More specifically we found:

1. HFPK 334 as a new bona-fide SNR, without an optical signature.

2. DEMS5 as a definite SNR with a very complex shell structure.

3. SNR B0050-72.8 as either an SNR or a supernova which exploded in a large bubble with a complex filamentary structure.

4. SNR B0058-71.8 an SNR with significantly smaller X-ray diameter not seen in any other known SNR.

All known SNRs in the SMC show a vast variety of types and environments in which they exist. Some SNRs could be confused with bubbles due to very complex and crowded fields. We find that the majority of the SMC's SNRs are in the adiabatic phase of their evolution with an average diameter of $45 \mathrm{pc}$.

We argue that high-resolution systematic X-ray, optical and radio-continuum observations of the SMC/LMC and other galaxies allow us to understand the overall multi-wavelength properties of SNRs.

Acknowledgements. The XMM-Newton project is supported by the Bundesministerium für Wirtschaft und Technologie/Deutsches Zentrum für Luft- und Raumfahrt (BMWI/DLR, FKZ 50 OX 0001) and the Max-Planck Society. We used the Karma/MIRIAD software packages developed by the ATNF. The Australia Telescope Compact Array is part of the Australia 
Telescope which is funded by the Commonwealth of Australia for operation as a National Facility managed by the CSIRO. MDF would like to thank the Max-Planck-Institut für extraterrestrische Physik for support. P.F.W. acknowledges the many contributions of R.C. Smith of CTIO and other members of the MCELS team, and financial support from US National Science Foundation through grant AST-0307613. B.E.R. acknowledges support from the US National Science Foundation through grant AST-0353997 to the Keck Northeast Astronomy Consortium REU program. We thank the referee for their excellent comments that improved this manuscript.

\section{References}

Borkowski, K. J., Lyerly, W. J., \& Reynolds, S. P. 2001, ApJ, 548, 820 Brück, M. T. 1976, Occasional Reports of the Royal Observatory Edinburgh, 1 Davies, R. D., Elliott, K. H., \& Meaburn, J. 1976, MmRAS, 81, 89

Dickel, J. R., Williams, R. M., Carter, L. M., et al. 2001, AJ, 122, 849

Filipović, M. D., Bohlsen, T., Reid, W., et al. 2002, MNRAS, 335, 1085 Filipović, M. D., Payne, J. L., Reid, W., et al. 2005, MNRAS, 364, 217

Gaensler, B. M., Hendrick, S. P., Reynolds, S. P., \& Borkowski, K. J. 2003, ApJ, 594, L111

Gooch, R. 2006, Karma Users Manual (Australia Telescope National Facility) Haberl, F., \& Pietsch, W. 2007 [arXiv:0712 .2720]

Haberl, F., Filipović, M. D., Pietsch, W., \& Kahabka, P. 2000, A\&AS, 142, 41

Hilditch, R. W., Howarth, I. D., \& Harries, T. J. 2005, MNRAS, 357, 304

Høg, E., Fabricius, C., Makarov, V. V., et al. 2000, A\&A, 355, L27
Hughes, J. P., \& Smith, R. C. 1994, AJ, 107, 1363

Jansen, F., Lumb, D., Altieri, B., et al. 2001, A\&A, 365, L1

Mathewson, D. S., Ford, V. L., Dopita, M. A., et al. 1984, ApJS, 55, 189

Mills, B. Y., Little, A. G., Durdin, J. M., \& Kesteven, M. J. 1982, MNRAS, 200, 1007

Nazé, Y., Manfroid, J., Stevens, I. R., Corcoran, M. F., \& Flores, A. 2004, ApJ, 608, 208

Pannuti, T. G., Schlegel, E. M., \& Lacey, C. K. 2007, AJ, 133, 1361

Parker, Q. A., Phillipps, S., Pierce, M. J., et al. 2005, MNRAS, 362, 689

Payne, J. L., Filipović, M. D., Pannuti, T. G., et al. 2004, A\&A, 425, 443

Payne, J. L., White, G. L., Filipović, M. D., \& Pannuti, T. G. 2007, MNRAS, 376, 1793

Reid, W. A., Payne, J. L., Filipović, M. D., et al. 2006, MNRAS, 367, 1379

Russell, S. C., \& Dopita, M. A. 1992, ApJ, 384, 508

Sasaki, M., Haberl, F., \& Pietsch, W. 2002, A\&A, 392, 103

Sault, B., \& Killeen, N. 2006, Miriad Users Guide (Australia Telescope National Facility)

Smith, C., Points, S., \& Winkler, P. F. 2006, NOAO Newsletter, 85, 6

Strüder, L., Briel, U., Dennerl, K., et al. 2001, A\&A, 365, L18

Turner, M. J. L., Abbey, A., Arnaud, M., et al. 2001, A\&A, 365, L27

Turtle, A. J., Ye, T., Amy, S. W., \& Nicholls, J. 1998, PASA, 15, 280

van der Heyden, K. J., Bleeker, J. A. M., \& Kaastra, J. S. 2004, A\&A, 421, 1031

Wang, Q., \& Wu, X. 1992, ApJS, 78, 391

Wilms, J., Allen, A., \& McCray, R. 2000, ApJ, 542, 914

Yokogawa, J., Imanishi, K., Tsujimoto, M., Koyama, K., \& Nishiuchi, M. 2003, PASJ, 55, 161 